\title{
СРК-подобные проявления
}

\section{ПРИ ДИВЕРТИКУЛЯРНОЙ БОЛЕЗНИ ТОЛСТОЙ КИШКИ}

\author{
В.М. МАХОВ ${ }^{1}$, Л.В. РОМАСЕНКО ${ }^{2}$, А.С. ПАНФЕРОВ ${ }^{1}$, Ю.А. ДОРОНИНА ${ }^{1}$, А.А. БАЛАХОНОВ ${ }^{1}$, М.В. ЮРАЖ ${ }^{1}$ \\ ${ }^{1}$ Федеральное государственное автономное образовательное учреждение высшего образования «Первый Московский государствен- \\ ный медицинский университет имени И.М. Сеченова» Министерства здравоохранения Российской Федерации (Сеченовский универси- \\ тет): 119991, Россия, г. Москва, ул. Трубецкая, д. 8, стр. 2 \\ 2 Федеральное государственное бюджетное учреждение «Национальный медицинский исследовательский центр психиатрии и нарко- \\ логии им. В.П. Сербского» Министерства здравоохранения Российской Федерации: 119034, Россия, г. Москва, Кропоткинский пер., д. 23
}

\section{Информация об авторах:}

Махов Валерий Михайлович - д.м.Н., профессор, научный руководитель гастроэнтерологического отделения Клиники факультетской терапии имени В.Н. Виноградова Федерального государственного автономного образовательного учреждения высшего образования «Первый Московский государственный медицинский университет имени И.М. Сеченова» Министерства здравоохранения Российской Федерации (Сеченовский университет), профессор кафедры факультетской терапии №1 лечебного факультета Федерального государственного автономного образовательного учреждения высшего образования «Первый Московский государственный медицинский университет имени И.М. Сеченова» Министерства здравоохранения Российской Федерации (Сеченовский университет); тел.: +7 (499) 245-43-02

Ромасенко Любовь Владимировна - Д.М.Н., профессор, руководитель отделения психосоматических расстройств Федерального государственного бюджетного учреждения
«Национальный медицинский исследовательский центр психиатрии и наркологии им. В.П. Сербского» Министерства здравоохранения Российской Федерации; тел.: +7(499) 248-75-96; e-mail: tromasenko@ mail.ru

Панферов Александр Сергеевич - к.м.Н., доцент кафедры факультетской терапии №1 Федерального государственного автономного образовательного учреждения высшего образования «Первый

Московский государственный медицинский университет имени И.М. Сеченова» Министерства здравоохранения Российской Федерации (Сеченовский университет); тел.: +7(916) 612-68-65; e-mail: a_panferov@mail.ru

Доронина Юлия Андреевна - аспирант кафедры факультетской терапии №1 Федерального государственного автономного образовательного учреждения высшего образования «Первый Московский государственный медицинский университет имени И.М. Сеченова» Министерства здра- воохранения Российской Федерации (Сеченовский университет); тел.: +7(916) 084-81-64

Балахонов Александр Алексеевич - аспирант кафедры факультетской терапии №1 Федерального государственного автономного образовательного учреждения высшего образования «Первый Московский государственный медицинский университет имени И.М. Сеченова» Министерства здравоохранения Российской Федерации (Сеченовский университет); тел.: +7(926) 706-60-18

Юраж Марта Валерьевна - врачгастроэнтеролог гастроэнтерологического отделения Клиники факультетской терапии имени В.Н. Виноградова Федерального государственного автономного образовательного учреждения высшего образования «Первый Московский государственный медицинский университет имени И.М. Сеченова» Министерства здравоохранения Российской Федерации (Сеченовский университет); тел.: +7(916) 178-18-49

\section{PEBЮME}

Статья посвящена дивертикулярной болезни толстой кишки, ее неосложненной форме, связи с возрастом. Освещены симптомы, позволяющие определить наличие при этой форме болезни СРК-подобных проявлений - болей и нарушений психоэмоционального статуса. Представлены результаты психометрического исследования и консультации психиатра 102 больных. Освещена роль ожирения в генезе дивертикулярной болезни. Представлено значение спазмолитической терапии, в частности мебеверина.

Ключевые слова: дивертикулярная болезнь толстой кишки, СРК-подобные проявления, психический статус, спазмолитики, мебеверин

Для цитирования: Махов В.М., Ромасенко Л.В., Панферов А.С., Доронина Ю.А, Балахонов А.А., Юраж М.В. СРК-подобные проявления при дивертикулярной болезни толстой кишки. Медицинский совет. 2019; 3: 130-135. DOI: https://doi.org/10.21518/2079-701X-2019-3-130-135.

Конфликт интересов: авторы заявляют об отсутствии конфликта интересов

\section{IBS-like manifestations}

\section{IN LARGE INTESTINE DIVERTICULAR DISEASE}

\author{
Valery M. MAKHOV ${ }^{1}$, Lyubov V. ROMASENKO르, Alexander S. PANFEROV ${ }^{1}$, Yulia A. DORONINA ${ }^{1}$, Alexander A. BALAKHONOV ${ }^{1}$, \\ MartaV. YURAZH ${ }^{1}$ \\ ${ }^{1}$ Federal State Autonomous Educational Institution of Higher Education «First Moscow State Medical University named after I.M. Sechenov» \\ of the Ministry of Health of the Russian Federation (Sechenov University): 119991, Russia, Moscow, Trubetskaya Street, 8, bldg. 2 \\ 2 Federal State Budgetary Institution «National Medical Research Center for Psychiatry and Narcology named after V.P. Serbsky» of the \\ Ministry of Health of the Russian Federation: 119034, Russia, Moscow, Kropotkinskiy lane, 23
}

\section{Author credentials:}

Makhov Valery Mikhailovich - Dr. of Sci. (Med), Professor, Scientific Director of the Gastroenterology Department of the Department of Faculty Therapuy of
Vinogradov Clinic of the Federal State Autonomous Educational Institution of Higher Education «First Moscow State Medical University named after I.M.
Sechenovy of the Ministry of Health of the Russian Federation (Sechenov University), Professor of the Department of Faculty Therapy No. 1 of the Faculty of Medicine of 
the Federal State Autonomous Educational Institution of Higher Education "First Moscow State Medical University named after I.M. Sechenov» of the Ministry of Health of the Russian Federation (Sechenov University); tel: +7 (499) 245-43-02

Romasenko Lyubov Vladimirovna - Dr. of Sci. (Med), Professor, Head of the Psychosomatic Disorders Department of the Federal State Budgetary Institution «National Medical Research Center of Psychiatry and Narcology named after V.P. Serbsky». tel: +7(499) 248-

75-96; e-mail: Iromasenko@mail.ru

Panferov Alexander Sergeevich - Cand. of Sci. (Med), Associate Professor of the Department of Faculty Therapy No.1 of the Federal State
Autonomous Educational Institution of Higher Education «First Moscow State Medical University named after I.M. Sechenov» of the Ministry of Health of the Russian Federation (Sechenov University); tel: +7(916) 612-68-65; e-mail: a_panferov@mail.ru Doronina Yulia Andreevna - Postgraduate Student of the Department of Faculty Therapy No.1 of the Federal State Autonomous Educational Institution of Higher Education «First Moscow State Medical University named after I.M. Sechenov» of the Ministry of Health of the Russian Federation (Sechenov University); tel: +7(916) 084-81-64 Balakhonov Alexander Alekseevich Postgraduate student of the Department of
Faculty Therapy No.1 of the Federal State Autonomous Educational Institution of Higher Education «First Moscow State Medical University named after I.M. Sechenov» of the Ministry of Health of the Russian Federation (Sechenov University); tel: $+7(926)$ 706-60-18

Yurazh Marta Valeryevna - Gastroenterologist of the Department of Gastroenterology of Vinogradov Clinic of the Faculty Therapy of the Federal State Autonomous Educational Institution of Higher Education «First Moscow State Medical University named after I.M. Sechenov» of the Ministry of Health of the Russian Federation (Sechenov University); tel: +7(916) 178-18-49

\section{ABSTRACT}

The article is devoted to the diverticular colon disease, its uncomplicated form, the connection with age. Symptoms allowing to define presence at this form of disease of IBS-like symptoms - pains and infringements of the psycho-emotional status are highlighted. The results of psychometric research and psychiatrist's consultation of 102 patients are presented. The role of obesity in the genesis of diverticular disease is highlighted. The significance of spasmolytic therapy, in particular mebeverine, is presented.

Keywords: colon diverticular disease, IBS-like manifestations, mental status, spasmolytics, mebeverine

For citing: Makhov V.M., Romasenko H.V., Panferov A.S., Doronina Yu.A., Balakhonov A.A., Yurazh M.V. IBS-like manifestations in large intestine diverticular disease. Meditsinsky Sovet. 2019; 3: 130-135. DOI: https://doi.org/10.21518/2079-701X-2019-3-130-135.

Conflict of interest: The authors declare no conflict of interest.

ивертикулы - грыжевые выпячивания стенки толстой кишки - самые частые дефекты, выявляемые при ирригоскопии и колоноскопии. Состояние, при котором в толстой кишке имеется хотя бы один дивертикул, определяется как дивертикулез ободочной кишки (ДОК). При ДОК дивертикулы по происхождению приобретенные, по строению - ложные, так как в их стенке отсутствуют мышечные волокна и подслизистый слой, по морфофункциональным особенностям - пульсионные, то есть возникшие вследствие продолжительного внутрикишечного давления на стенку кишки. Преобладающая локализация дивертикулеза у европеоидов левая часть ободочной кишки.

Точных данных о распространенности ДОК нет, что в значительной мере связано со сложностью и высокой стоимостью инструментальной диагностики. Бесспорно нарастание выявляемости ДОК с увеличением возраста: ДОК выявляют у 5-10\% в возрасте 40-50 лет, до 30\% - в группе старше 60 лет и у 64-65\% обследованных старше 80 лет [1].

Среди факторов, предполагаемых как «факторы риска» ДОК, на первом месте рассматривается пищевой рацион с низким содержанием растительной клетчатки (пищевых волокон), их недостаток приводит к уменьшению объема кала, повышению его плотности, что в сочетании с возникающим вслед за этим запором и другой дисмоторикой приводит к повышению давления в полости толстой кишки. В зонах повышенного внутриполостного давления и происходит пролабирование слизистой оболочки в «слабых» местах прохождения через нее сосудов. Недостаток пищевых волокон (клетчатки) приводит также к избыточному бактериальному росту, изменению эластического состояния кишки, негативно влия- ет на рецепторный аппарат и интрамуральные ганглии, что ведет к хаотизации моторики и повышению внутрикишечного давления. Доказательством роли питания служат наблюдения за лицами, соблюдающими вегетарианство, у которых и дивертикулез, и осложненную ДБ наблюдают гораздо реже. Обсуждается роль ожирения, главным образом абдоминального, висцерального в генезе ДОК.

Употребление красного мяса и курение оказывают на генез ДОК незначительное влияние, негативная роль алкоголя, кофеина, орехов не доказана.

В отличие от ДОК - понятия скорее анатомического, дивертикулярную болезнь толстой кишки (ДБТК) определяют как заболевание, характеризующееся клиническими проявлениями воспалительного процесса и его возможными осложнениями [1]. В таком случае остается большая группа пациентов, у которых нет клинически значимого воспаления, но существуют различные нарушения функции. Примерно у 80\% пациентов ДОК протекает без осложнений [2].

В сферу внимания врачей-терапевтов, и главным образом хирургов, прежде всего попадают пациенты ДОК с воспалением дивертикула и последующими осложнениями: перидивертикулитом, абсцессом, перфорацией, свищом, перитонитом [3].

Если понимать дивертикулярную болезнь (дивертикулез) толстой кишки как морфофункциональный патологический процесс, характерным отличительным признаком которого является наличие мешковидных выпячиваний стенки ободочной кишки (дивертикулов) [4], то любые клинические проявления, не только осложнения, должны быть оценены как обоснование терапии и профилактики осложнений. 
- Таблица 1. Классификационные признаки состояний, связанных с дивертикулами ободочной кишки

- Table 1. Classification of states associated with colon diverticulas

\begin{tabular}{l|l}
\multicolumn{1}{c|}{ Определение } & \multicolumn{1}{|c}{ Классификационные привнаки } \\
\hline $\begin{array}{l}\text { А.Дивертикулез } \\
\text { ободочной кишки }\end{array}$ & $\begin{array}{l}\text { 1. Наличие дивертикулов } \\
\text { 2. Отсутствие каких-либо симптомов, причиной } \\
\text { которых могут быть дивертикулы }\end{array}$ \\
\hline $\begin{array}{l}\text { Б. Клинически } \\
\text { выраженный } \\
\text { дивертикулез }\end{array}$ & $\begin{array}{l}\text { 1. Наличие дивертикулов } \\
\text { 2. Наличие клинической симптоматики, } \\
\text { происхождение которой может быть связано с } \\
\text { формированием дивертикулов в кишке } \\
\text { 3. Отсутстве признаков воспалительного процесса } \\
\text { или кровотечения, источником которых является } \\
\text { один из дивертикулов }\end{array}$ \\
\hline $\begin{array}{l}\text { В.Дивертикулярная } \\
\text { болезнь }\end{array}$ & $\begin{array}{l}\text { 1. Наличие дивертикулов } \\
\text { 2. Наличие воспалительного процесса или } \\
\text { кровотечения, источником которых является один } \\
\text { или несколько дивертикулов ободочной кишки }\end{array}$ \\
\hline
\end{tabular}

Ряд позиций классификации ДБТК до настоящего времени обсуждаются. Так, предложено выделить следующие формы ДБТК [3]:

а) бессимптомную форму ДБ (дивертикулез) - это дивертикулы толстой кишки без клинических проявлений;

б) неосложненную форму с клиническими проявлениями (клинически выраженный дивертикулез);

в) осложненную форму ДБ с воспалением и другими осложнениями, обусловленными дивертикулами.

В 2016 г. предложена следующая классификация (табл. 1) [1].

Часть лиц с ДОК жалоб не предъявляют, чаще всего адаптированы к регулированию стула, и, учитывая возраст, при их полиморбидности ДОК их не беспокоит. Другая часть становится пациентами с жалобами на боли, обычно левосторонние, чаще спастического характера по ходу толстой кишки, нарушение стула, чаще - запоры, вздутие.

Оценивая клиническую картину неосложненной формы ДБТК, можно отметить сходство таких проявлений, как боли, связанные с дефекацией и ассоциированные с изменением частоты и формы стула, вздутие с аналогичными клиническими проявлениями синдрома раздраженного кишечника (СРК) [3, 5]. Следует подчеркнуть, что речь идет о пациентах, у которых отсутствуют эндоскопические признаки дивертикулита и сегментарного колита. Если сравнить наблюдаемое при ДБТК с диагностическими критериями СРК («Рим IV»), то вывод о схожести симптомов однозначен.

Сравнение болей при СРК и ДБТК представлено в таблище 2.

При неосложненной форме ДБ (НФДБ), так же как и при СРК, в возникновении клинических симптомов большую роль играет висцеральная гиперчувствительность толстой кишки [6]. Существует даже мнение, что НФДБ это СРК на фоне дивертикулеза.

Ситуацию, когда при органической патологии толстой кишки возникают симптомы, схожие с СРК, обозначают как СРК-подобный синдром, или СРК-подобные проявления, или синдромом перекреста. При этом помимо аналогич- ных с СРК характеристик болей обращают внимание на схожесть в психовегетативном и эмоциональном статусе.

Признано, что СРК - биопсихосоциальное функциональное кишечное расстройство, в основе которого лежит сочетание психосоциального воздействия и сенсомоторной дисфункции. Отмечено, что сенсомоторные нарушения также играют роль в появлении и прогрессировании ДБТК.

Тщательный анализ клинической картины, сопоставление морфологического исследования с результатами ирригоскопии и колоноскопии у пациентов с ДБТК позволили авторам [7] выделить два клинико-морфологических варианта неосложненной ДБТК, влияющих на качество жизни: СРК-подобный вариант и ишемический вариант. Исследования продемонстрировали, что показатели психического здоровья при СРК-подобном варианте достоверно ниже средних значений, а в слизистой оболочке выявлено неспецифическое микроскопическое воспаление. При ишемическом варианте биопсия слизистой определила дистрофические процессы.

К факторам, которые можно рассматривать как способствующие СРК-подобным проявлениям при ДБТК, можно отнести: висцеральную гиперчувствительность, дискинезию толстой кишки, воспаление малой интенсивности, повышение провоспалительных цитокинов, расстройство взаимодействия «ЖКТ - мозГ» (disordersofgut-braininteraction).

У части пациентов ДБТК возможно выявление лабораторных признаков, характерных для постинфекционного СРК: в анамнезе - ОКИ, антитела к возбудителю ОКИ в крови в низких титрах, маркеры ОКИ в кале, изменения фекальной микрофлоры, высокий бактериальный рост в тонкой кишке, снижение показателей иммунитета, положительный эффект антибактериальной терапии.

Мы исследовали некоторые факторы, которые могли позволить оценить СРК-подобные проявления при ДБТК.

Обследованы 102 пациента с ДБТК (28,5\% мужчин и $71,5 \%$ женщин) в возрасте: от 40 до 50 лет - 8 человек (7,8\%), от 51 до 60 лет - 45 человек (44,1\%), от 61 до 70 лет - 30 человек (29,4\%), старше 70 лет - 19 человек $(18,6 \%)$.

Важнейшим моментом, во многом влияющим на общее состояние больного, а также на результаты лечения и прогноз, на качество жизни, является психический статус пациента. Особо важно своевременное выявление

Таблица 2. Болевой синдром при СРК и ДБТК

Table 2. Pain syndrome in IBS and DCD

\begin{tabular}{l|l}
\multicolumn{2}{c}{ Схожие проявления } \\
\hline $\begin{array}{l}\text { - Усиление перед позывом на дефекацию } \\
\text { - Облегчение после дефекации } \\
\text { - Отсутствие боли ночью }\end{array}$ \\
\hline \multicolumn{1}{|c|}{ Р Различия } \\
\hline \multicolumn{1}{|c|}{ Соль в животе без четкой } \\
\begin{tabular}{l|l} 
локализации \\
\hline Боль кратковременная
\end{tabular} & $\begin{array}{l}\text { Четкая локализация, чаще в левой } \\
\text { подвздошной области }\end{array}$ \\
\hline
\end{tabular}


депрессивных расстройств. Депрессия может увеличивать риск негативного прогноза прежде всего через изменение поведения, включая отказ от инвазивных вмешательств, пренебрежение медикаментами и диетой. Депрессия сопровождается повышением в крови провоспалительного цитокина ФНО-а, что может влиять на состояние слизистой оболочки толстой кишки.

Результаты психометрического исследования и консультации психиатра представлены в таблице 3.

Как видно из таблицы, только у 12 пациентов (11,7\%) отклонений в психическом статусе не выявлено. Преобладают тревожно-депрессивные, ипохондрические и тревожно-фобические расстройства. На консультацию психиатра пациенты были направлены в связи с тревожными расстройствами и ипохондрическими страхами возможности запоров, «тяжелой интоксикации» в связи с запорами, а также канцерофобическими переживаниями, некупирующимися болями по ходу кишечника. При ДБТК пациенты имели гастроэнтерологический анамнез длительностью от 2 до 10 лет; их лечили по поводу синдрома раздраженного кишечника с различными клиническими вариантами. Все пациенты относились к старшей возрастной группе, преобладали больные старше 65 лет. У части пациентов имели место анатомические особенности строения кишечника в виде долихосигмы.

Расстройства в большинстве наблюдений выявлялись значительно раньше соматического заболевания - в юношеском и молодом возрасте (рекуррентные депрессивные расстройства, циклотимия, личностная патология), соматогенные и психогенные психические расстройства носили симптоматический характер на фоне обострения соматического заболевания либо представляли собой соматогенные проявления психической дезадаптации, кроме того, дополнялись психическими расстройствами в рамках реакций личности на болезнь. Во всех этих случаях задачей консультанта-психиатра было оказание психотерапевтической поддержки и медикаментозной помощи для адекватной адаптации пациента к ситуации, связанной с болезнью. В ряде случаев врач-психиатр являлся связующим звеном между пациентом и врачом-гастроэнтерологом в связи с двойным влиянием соматической болезни на психику человека: соматогенным и психогенным.

В случаях клинически завершенных тревожно-депрессивных, тревожно-фобических синдромов применяли малые дозы антидепрессантов, преимущественно из группы СИОЗС, нейролептики в малых дозах: Сульпирид 100 мг/сут, Сонапакс 10-15 мг/сут, Этаперазин 2-3 мг/сут, а также транквилизаторы: Феназепам 1 мг/сут, этифоксин (Стрезам) 100 мг/сут.

Похожая структура психических отклонений была выявлена при СРК [8].

Мы обратили внимание на то, что более чем у 2/3 обследованных больных была повышена масса тела: нормальная масса тела наблюдалась у 28 пациентов (27,4\%), избыточная масса тела - у 57 больных (55,8\%), ожирение 1-й степени - у 14 человек (13,7\%), ожирение 2-й степени - у 3 (2,9\%). По мере нарастания ИМТ отмечено усиление болевого синдрома, усугубление запора, нарастание
Таблица 3. Результаты психометрического исследования и консультации психиатра $(\mathrm{n}=102)$

Table 3. Results of psychometric study and psychiatrist consultation $(n=102)$

\begin{tabular}{l|c|c}
\multicolumn{1}{c|}{ Выявленные расстройства } & $\begin{array}{c}\text { Количество } \\
\text { пациентов }\end{array}$ & $\%$ \\
\hline $\begin{array}{l}\text { признаков психической дезадаптации не выявлено } \\
\text { тревожно-депрессивное и ипохондрическое рас- } \\
\text { стройства }\end{array}$ & 12 & 11,7 \\
\hline тревожно-фобическое расстройство & 27 & 33,3 \\
\hline депрессивное расстройство & 14 & 13,7 \\
\hline генерализованное тревожное расстройство & 8 & 7,8 \\
\hline невротические расстройства & 5 & 4,9 \\
\hline Инсомническое расстройство & 2 & 1,9
\end{tabular}

тяжести психических девиаций. Связь избыточной массы тела, ожирения и ДОК отслежена в ряде исследований.

Метаанализ 6 проспективных исследований, включавших в себя более полутора миллионов участников, среди которых зарегистрировано 28915 случаев дивертикулеза, продемонстрировал линейную зависимость между индексом массы тела и риском возникновения ДБТК.Увеличение индекса массы тела с 20 кг/м² на каждые 5 единиц сопровождается ростом относительного риска развития ДБТК на 28\%, достигая 2-3-кратного увеличения у лиц с ожирением. Также продемонстрировано, что физическая активность снижает риск дивертикулеза и дивертикулита на 24 и 26\% соответственно [9].

Среди пациентов с ДБТК отмечается повышенное содержание общего, висцерального и подкожного жира по сравнению с контрольной группой, при этом соотношение висцерального и подкожного жира между группами не различается [10].

Продемонстрировано увеличение площади висцерального жира у пациентов с левосторонним дивертикулитом по сравнению с группой пациентов с дивертикулезом [11]. Среди пациентов, подвергшихся экстренному хирургическому вмешательству по поводу дивертикулита, достоверно выше соотношение висцерального и подкожного жира по данным КТ. Вероятно, более напряженный провоспалительный фон у пациентов с висцеральным ожирением может способствовать более тяжелому течению ДБТК [12].

Ведущей жалобой пациентов, обратившихся в клинику, были боли, локализующиеся в левой половине живота, чаще в левой подвздошной области. Боли имели спастический, сжимающий характер, не иррадиировали, сопровождались вздутием.

Только 6 человек из 102 обследованных не предъявляли жалоб на боли. У 72 (72,5\%) боли были связаны с запорами или поносами, протекали по типу кишечных колик, у 22 боль облегчалась после дефекации. Таким образом, можно отметить, что, как и при СРК, в генезе боло нарушение моторной функции кишечника - гипер- 
моторная спастическая дискинезия, предполагающая назначение спазмолитиков.

Можно считать, что применение селективных спазмолитиков при ДБТК патогенетически обосновано, так как включает следующие механизмы:

- Уменьшает/ликвидирует сегментарный спазм мускулатуры толстой кишки и облегчает/ликвидирует болевой синдром.

- Снижает внутрипросветное давление.

- Уменьшает транслокацию микроорганизмов в слизистой толстой кишки.

- Уменьшает возможность клапанного попадания и уплотнения кишечного содержимого в дивертикул.

- Снижает тонус «шейки» дивертикула.

Рекомендации РГА и АК России (2016) декларируют: лечение пациентов с данной формой заболевания (клинически выраженным дивертикулезом) не отличается от лечения синдрома раздраженного кишечника, проводится в течение длительного времени с обязательным учетом индивидуальных особенностей и включает коррекцию диеты и назначение селективных спазмолитиков.

Используемые в гастроэнтерологии спазмолитики относят к нейротропным и миотропным.

1. К первым относят препараты, влияющие на проведение нервного импульса (нейротропные спазмолитики) холинолитические средства (атропин, гиосцинабутилбромид и др.); нейротропные спазмолитики с множественным механизмом действия (тримебутина малеат).

2. Вторую группу составляют препараты, влияющие прямо на гладкомышечную клетку (миотропные спазмолитики): блокаторы натриевых каналов (мебеверина гидрохлорид); блокаторы кальциевых каналов (пинаверия бромид, отилония бромид); ингибиторы фосфодиэстеразы ФДЭ4 (папаверина гидрохлорид, дротаверина гидрохлорид); донаторы оксида азота (нитраты).

Спазмолитик мебеверин на протяжении многих лет успешно используют при дискинетических, спастических расстройствах кишечника и билиарного тракта. Мебеверин блокирует работу быстрых натриевых каналов клеточной мембраны миоцита, что нарушает процессы поступления натрия в клетку, замедляет процессы деполяризации и прекращает вход кальция в клетку через медленные каналы. В результате снимается спазм мышечного волокна. Снижение пополнения внутриклеточных кальциевых депо на фоне применения мебеверина приводит к кратковременному выходу ионов калия из клетки и ее гиперполяризации, что предупреждает развитие гипотонии мышечной стенки. Мебеверин устраняет спазм, не влияя на нормальную перистальтику кишечника, ЖП и сфинктера Одди, не оказывает антихолинергического эффекта. Препарат зарекомендовал себя как высокоэффективный спазмолитик и безопасное лекарственное средство.

Прием мебеверина в дозах, достигавших 800 мг/сут, курсами до 12 недель продемонстрировал высокую переносимость и безопасность $[13,14]$. Метаанализ исследований препаратов, используемых при СРК, показал, что мебеверин вызывает наименьшее количество побочных эффектов, отмечены только редкие кожные аллергические проявления и редкие явления слабости $[15,16]$.

Результаты терапии мебеверином в течение 4 недель, как показало многоцентровое исследование 218 больных с постхолецистэктомическими спазмами, позволяют рассматривать мебеверин как эффективное средство при абдоминальной боли, симптомах диспепсии и нарушении стула. Более выраженное улучшение отмечено при 6-недельном приеме препарата [17].

Позитивный эффект при ПХЭС достигается и благодаря воздействию мебеверина и на толстую кишку.

Мебеверин - селективный миотропный спазмолитик, препарат выбора при спазмах, связанных с билиарной или панкреатической патологией. Эффективно устраняет клинические проявления: боль и тяжесть в правом подреберье, тошноту, горечь во рту, обладает высокой тропностью к сфинктеру Одди, нормализует моторику двенадцатиперстной кишки, снижает дуоденальную гипертензию, дуоденогастральные и дуоденопанкреатические рефлюксы, нормализует отток желчи. Препарат действует избирательно на гладкомышечные клетки желудочно-кишечного тракта, устраняет спазмы без влияния на нормальную перистальтику кишечника. Препарат обладает двойным механизмом действия: блокирует $\mathrm{Na+-каналы} \mathrm{и} \mathrm{препятствует} \mathrm{развитию}$ спазма, блокирует Са++-депо, ограничивая выход К+ из клетки, препятствуя развитию гипотонии [18].

Достоинством мебеверина является очень высокая релаксирующая активность в отношении сфинктера Одди, в 20-40 раз превышающая таковую папаверина. При этом мебеверин оказывает нормализующее влияние на мускулатуру кишечника, устраняя функциональный дуоденостаз, гиперперистальтику, спазмофилию, не вызывая при этом нежелательной гипотонии [19].

При хроническом панкреатите, как правило, диагностируется функциональный спазм сфинктера Одди, дуоденальная гипертензия. В этом случае ключевую роль в лечении играют селективные спазмолитики, например мебеверин [20].

При беременности допускается использование мебеверина при положительном соотношении риска для плода и пользы для матери. Препарат избирательно действует на гладкую мускулатуру органов пищеварения, не влияя на тонус матки и сосуды $[21,22]$.

Кроме того, мебеверин расслабляет сфинктер Одди, который регулирует поступление желчи и поджелудочного сока в двенадцатиперстную кишку. Поскольку у беременных сфинктер Одди часто спазмирован, возникает спастическая боль. Препарат принимают по 200 мг 2 раза в сутки - за 20 мин до завтрака и до ужина [23].

Действие мебеверина развивается быстро (через 20-30 мин) и продолжается в пределах 12 часов, что делает возможным его двукратный прием в сутки (пролонгированная форма) [24].

Проведенные исследования позволили выявить ряд чрезвычайно важных отличительных особенностей действия мебеверина, позволяющих считать его препаратом первого выбора в терапии заболеваний с симптомами обусловленными спастической или гипермоторной дис- 
кинезией гладкой мускулатуры пищеварительного тракта: быстрое наступление терапевтического эффекта (препарат начинает действовать уже через 20-30 мин после приема);

- действие однократного приема препарата сохраняется на протяжении 12 ч;

сохранение достигнутой клинической ремиссии заболеваний в течение длительного времени после прекращения лечения (так, после окончания 8-недельного курса лечения мебеверином синдрома раздраженного кишечника (СРК) и функциональных расстройств билиарной системы положительный терапевтический эффект сохраняется 6 мес. и более); хорошая переносимость, отсутствие системных действий и побочных эффектов, требующих отмены препарата [25]. - Таким образом, в задачи лечения неосложненной формы ДБТК и профилактики осложнений входят:

- Облегчение болевого синдрома - спазмолитики (мебеверин).

- Изменение рациона питания (увеличение количества пищевых волокон до 25-26 г в день - пшеничные отруби 20-37 г/сут).

- Коррекция микрофлоры кишечника (рифаксимин, месалазин).

- Уменьшение избыточной массы тела.

Нормализация психического статуса.

Поступила/Received 17.03.2019

\section{MUTEPATYPA/REFERENCES}

1. Ивашкин В.Т., Шелыгин Ю.А., Ачкасов С.И. [и др.]. Рекомендации Российской гастроэнтерологической ассоциации и Ассоциации колопроктологов России по диагностике и лече нию взрослых больных дивертикулярной болезнью ободочной кишки. Российский жур нал гастроэнтерологии, гепатологии, колопроктологии. 2016;26(1):65-80. https://doi. org/10.22416/1382-4376-2016-26-1-65-80. [Ivashkin V.T., Shelygin Y.A., Achkasov S.I. [et al.] Recommendations of the Russian

Gastroenterological Association and the Association of Coloproctologists of Russia on diagnosis and treatment of adult patients with colon diverticular disease. Russian Journal of Gastroenterology, Hepatology, Coloproctology [Rossijskij zhurnal gastroenterologii, gepatologii, koloproktologii.]. 2016;26(1):65-80. https://doi. org/10.22416/1382-4376-2016-26-1-65-80.] (In Russ.)

2. Carabotti M., Annibale B. Treatment of diverticular disease: an update on latest evidence and clinical implications. Drugs Context. 2018;7:ID212526

3. Сабельникова Е.А. Актуальные вопросы лечения и профилактики дивертикулярной болезни. Эффективная фармакотерапия.

2018:16:38-43. [Sabelnikova E.A. Topical issues of treatment and prevention of diverticular disease. Effective pharmacotherapy [Effektivnaya farmakoterapiya]. 2018;16:38-43.] (In Russ.)

4. Орлова Л.П., Трубачева Ю.Л. Возможности современной ультразвуковой диагностики дивертикулеза ободочной кишки. Медицинская визуализация. 2010;1:128-129. [Orlova L.P., Trubacheva Y.L. Possibilities of modern ultrasonic diagnostics of colon diverticulosis. Medical imaging [Medicinskaya vizualizaciya.]. 2010;1:128-129.] (In Russ.)

5. Cremon C., Bellacosa L., Barbaro M.R. et al. Diagnostic challenges of symptomatic uncomplicated diverticular disease. Minerva Gastroenterol. Dietol. 2017;63(2):119-129.

6. Кукес В.Г. и др. Проблемы современной спазмолитической терапии в гастроэнтерологии. Медицинский алфавит. 2016;3(24):5-12. [Kukes V.G. et al. Problems of modern spasmolytic therapy in gastroenterology. Medical alphabet [Medicinskij alfavit]. 2016;3(24):5-12.] (In Russ.)

7. Левченко С.В.и др. Клинико-морфологические варианты дивертикулярной болезни толстой кишки. Экспериментальная и клиническая гастроэнтерология. 2013;3. [Levchenko S.V. et al. Clinical and morphological variants of colon diverticular disease. Experimental and clinical gastroenterology. [Eksperimental'naya i klinicheskaya gastroenterologiya.] 2013;3.] (In Russ.)
8. Махов В.М.и др. Синдром раздраженного кишечника - коморбидное соматопсихическое заболевание. РМЖ. Прил. к матер. конгр. Человек и лекарство. 2012;2. [Makhov V.M. et al. Irritated bowel syndrome - comorbid somatopsychological disease. RMJ. Attach. to the mater. of the Congress Human and Drugs [RMJ. Pril. k mater. kongr. Chelovek i lekarstvo.]. 2012;2. (In Russ.)] (In Russ.)

9. Aune D., Sen A., Leitzmann M.F., Norat T., Tonstad S., Vatten L.J. Body mass index and physical activity and the risk of diverticula disease: a systematic review and meta-analysis of prospective studies. Eur J Nutr. 2017;56:2423-2438. doi: 10.1007/s00394-0171443-x.

10. Lee S.P., Ahn Y.W., Lee O.Y., Lee K.N., Sohn W., Lee H.L., Jun D.W., Yoon B. Ch. Choi H.S. The relationship between colonic diverticulosis and abdominal visceral and subcutaneous fat accumulation measured by abdominal CT scan. Turk J Gastroenterol. 2014;25:192-7. doi: 10.5152/ tjg.2014.4581.

11. Yamada E., Ohkubo H., Higurashi T., et al. Visceral obesity as a risk factor for left-sided diverticulitis in Japan: a multicenter retrospective study. Gut Liver. 2013;7:532-538.

12. Docimo S.Jr., Lee Y., Chatani P., et al. Visceral to subcutaneous fat ratio predicts acuity of diverticulitis. Surg Endosc. 2017;31:2808-2812.

13. Kastein Eth. A double blind cross-over study of Serc (betahistine dihydrochloride), 36 mg daily, versus placebo in outpatients with vertigo. Duphar report H108.5009, 1983.

14. Van Outryve M., Mayeur S., Meeus M.A., Rosillon D., Hendrick B., Ceuppens M. J. Clin. Pharm. Ther. 1995;20:277-282.

15. Агафонова Н.А. и др. Эффективность мебеверина гидрохлорида в коррекции моторных нарушений кишечника. РМЖ. Болезни органов пищеварения. 2005;7(2):101. [Agafonova N.A. et al. Effectiveness of mebeverine hydrochloride in the correction of intestinal motor disorders. $R M J$. Diseases of the digestive system [RMZh. Bolezni organov pishchevareniya.] 2005;7(2):101.] (In Russ.)

16. Poynard T., Naveau S., Mory B., Chaput J.C. Metaanalysis of smooth muscle relaxants in the treatment of irritable bowel syndrome. Aliment. Pharmacol. Ther. 1994:8:499-510.

17. Маев И.В. и др. Результаты проспективной наблюдательной программы «Одиссей»: эффективность мебеверина у пациентов с постхолецистэктомическими спазмами Терапевтический архив. 2018;90(8):40-47. [Maev I.V. et al. Results of the prospective observation programme «Odyssey»: effective- ness of mebeverine in patients with postcholecystectomic spasms. Therapeutic archive [Terapevticheskij arhiv.]. 2018;90(8):40-47.] (In Russ.)

18. Ливзан М.А., Лялюкова Е.А. Патоморфоз хро нического панкреатита: новое в привычном (по материалам Европейского консенсуса 2013). Лечащий врач. [Lechashchij vrach.] 2014;8:1-9. (In Russ.)

19. Ардатская М.Д. Функциональные расстройства билиарного тракта: проблемы диагностики и лечения. Фарматека. 2012:2:71-77. [Ardatskaya M.D. Functional disorders of the biliary tract: problems of diagnosis and treatment. Pharmacy [Farmateka]. 2012;2:71-77.] (In Russ.)

20. Маев И.В., Дичева Д.Т., Андреев Д.Н. Актуальные аспекты патогенетической фармакотерапии хронического билиарнозависимого панкреатита. Эффективная фармакотерапия. 2012;26:12-17. [Maev I.V., Dicheva D.T., Andreev D.N. Actual aspects of pathogenetic pharmacotherapy of chronic biliary pancreatitis. Effective pharmacotherapy [Effektivnaya farmakoterapiya.]. 2012;26:12-17.] (In Russ.)

21. Кирющенков А.П. Влияние вредных факторов на плод. М.: Медицина, 1978. [Kiryushchenkov A.P. Influence of harmful factors on the fetus. M.: Medicine, 1978.] (In Russ.)

22. Еремина Е.Ю. Заболевания органов пищеварительной системы у беременных. Гастроэнтерология Санкт-Петербурга. 2011;4:2-6. [Eremina E.Yu. Diseases of the digestive system in pregnant women. Gastroenterology of St. Petersburg [Gastroenterologiya Sankt-Peterburga.]. 2011;4:2-6.] (In Russ.) 23. Циммерман Я.С., Михалева Е.Н. Возможности фармакотерапии при лечении гастроэнтерологических заболеваний в период беременности. Клиническая медицина. 2015;93:8. [Zimmerman Y.S., Mikhaleva E.N. Possibilities of pharmacotherapy in the treatment of gastroenterological diseases during pregnancy. Clinical medicine [Klinicheskaya medicina.]. 2015;93:8.] (In Russ.)

24. Маев И.В., Самсонов А.А., Андреев Н.Г. Болевой абдоминальный синдром и место спазмолитиков в его коррекции. Фарматека. 2012;11. [Maev I.V., Samsonov A.A., Andreev N.G. Abdominal pain syndrome and the place of spasmolytics in its correction. Pharmacy [Farmateka]. 2012;11.] (In Russ.)

25. Селиверстов П. и др. Функциональные расстройства билиарного тракта и их лечение. Врач. 2012;3:9-9. [Seliverstov P. et al. Functional disorders of the biliary tract and their treatment. Doctor [Vrach]. 2012;3:9-9.] (In Russ.) 